S. Huckaba and T. Marley

Nagoya Math. J.

Vol. 133 (1994), 57-69

\title{
DEPTH FORMULAS FOR CERTAIN GRADED RINGS ASSOCIATED TO AN IDEAL
}

\author{
SAM HUCKABA * AND THOMAS MARLEY**
}

\section{Introduction}

In this paper, we investigate the relationship between the depths of the Rees algebra $R[I t]$ and the associated graded ring $\operatorname{gr}_{I}(R)$ of an ideal $I$ in a local ring $(R, m)$ of dimension $d>0$. Here

$$
R[I t]:=R \oplus I t \oplus I^{2} t^{2} \oplus \cdots
$$

and

$$
\operatorname{gr}_{I}(R):=R / I \oplus I / I^{2} \oplus I^{2} / I^{3} \oplus \cdots
$$

These rings are important not only algebraically, but geometrically as well. For instance, Proj $R[I t]$ is the blow-up of $\operatorname{Spec}(R)$ with respect to $I$. The relationship between the Cohen-Macaulayness of these two rings has been studied extensively. We list here a few of the many results on this relationship:

(1.1) $R[I t]$ is Cohen-Macaulay for every parameter ideal $I$ of $R$ if and only if $R$ is Buchsbaum and $H_{m}^{i}(R)=0$ for $i \neq 1, d$ ([GS1]).

(1.2) Suppose $R$ is Cohen-Macaulay with infinite residue field and let $I$ be an $m$-primary ideal of $R$. Then $R[I t]$ is Cohen-Macaulay if and only if $\operatorname{gr}_{I}(R)$ is Cohen-Macaulay and the reduction number of $I$ is less than $d$ ([GS2]).

(1.3) Let $I$ be an ideal of $R$ of positive height. Then $R[I t]$ is Cohen-Macaulay if and only if $H_{N}^{i}\left(\operatorname{gr}_{I}(R)\right)_{n}=0$ for $n \neq-1$ and $i<d$, and $H_{N}^{d}\left(\operatorname{gr}_{I}(R)\right)_{n}=0$ for $n \geq 0$, where $N$ denotes the homogeneous maximal ideal of $\operatorname{gr}_{I}(R)$ ([TI]).

These theorems provide necessary and sufficient conditions for depth $R[I t]$ to be at its maximum. However, very little has been written concerning the relationship between depth $R[I t]$ and depth $\operatorname{gr}_{I}(R)$ in more generality. Schenzel touched on this topic in [Sch2] and [Sch3], in which he gave necessary and

Received October 19, 1992.

* The first author was partially supported by NSA grant MDA904-92-H-3040.

** The second author was partially supported by NSF grant DMS-9106739. 
sufficient conditions for certain elements to form a regular sequence in $R[I t]$. Also: in an earlier article, the present authors proved the following result:

(1.4) Suppose $R$ is Cohen-Macaulay and $I$ is an $m$-primary ideal. $\operatorname{If} \operatorname{gr}_{I}(R)$ is not Cohen-Macaulay then depth $R[I t]=\operatorname{depth} \operatorname{gr}_{I}(R)+1$ ([HM]).

(Here we adopt the convention that if $S$ is a Noetherian graded ring with a unique homogeneous maximal ideal $m$ then depth $S$ is defined to be $\operatorname{depth}_{m} S$.)

Thus, in the case $I$ is $m$-primary the only complication in relating the depths of $R[I t]$ and $\operatorname{gr}_{I}(R)$ occurs when depth $\operatorname{gr}_{I}(R)=\operatorname{dim} R$. The techniques we used to prove this result (induction on the depth of $\operatorname{gr}_{I}(R)$ ) do not extend to the case when $\operatorname{dim} R / I>0$, as we are not guaranteed the existence of a homogeneous regular element in $\operatorname{gr}_{I}(R)$ if depth $\operatorname{gr}_{I}(R)>0$. The question of whether or not (1.4) still holds if the $m$-primary assumption is removed was left unanswered. In this paper, we provide an affirmative answer to this question (Corollary 3.12). In addition, we prove the following:

Proposition 3.6. For any ideal $I$ of $R$, depth $R[I t] \geq \operatorname{depth} \operatorname{gr}_{I}(R)$.

Theorem 3.10. If depth $\operatorname{gr}_{I}(R)<\operatorname{depth} R$ then depth $R[I t]=$ depth $\operatorname{gr}_{I}(R)+1$.

Theorem 3.13. If $a_{s}\left(\operatorname{gr}_{I}(R)\right)<0$ then depth $R[I t] \geq \operatorname{depth} \operatorname{gr}_{I}(R)+1$, where $s=$ depth $\operatorname{gr}_{I}(R)$.

The methods employed in the proofs of these results differ from those used in [HM]. In place of induction, we make frequent use of graded local cohomology in a manner inspired by the works of Trung and Ikeda [TI] and Brodmann [Br1-3]. In [TI], the concept of generalized Cohen-Macaulayness is used to prove (1.3) above. In this paper, we stretch this idea further and introduce the notion of generalized depth. This concept is developed in Section 2 and plays a key role in our demonstrations of the above results. These proofs, along with several examples and an application to ideals generated by systems of parameters in Buchsbaum rings, are given in Section 3.

Throughout this paper, all rings are assumed to be commutative with identity and Noetherian. As a general reference, we refer the reader to [Mat] for any unexplained notation or terminology.

By $\operatorname{dim} R$ we always mean the Krull dimension of $R$. It is well-known that if $I$ is an ideal of $R$ then $\operatorname{dim} \operatorname{gr}_{I}(R)=\operatorname{dim} R$. If $I$ is not contained in some prime ideal of maximal dimension then $\operatorname{dim} R[I t]=\operatorname{dim} R+1$. (See [V] for proofs of 
these facts.) We remark here that $\operatorname{gr}_{I}(R) \cong R[I t] / I R[I t]$ as graded rings.

If $R$ is local with maximal ideal $m$ and $I$ is an ideal of $R$, the analytic spread of $I$, denoted $l(I)$, is defined to be $\operatorname{dim} R[I t] / m R[I t]$. In [NR], it is shown that ht $(I) \leq l(I) \leq \operatorname{dim} R$. An ideal $I$ is called equimultiple if $l(I)=$ ht $I$. In particular, all $m$-primary ideals are equimultiple. If $R / m$ is infinite and $l=l(I)$ then there exist elements $x_{1}, \ldots, x_{l} \in I$ such that $\left(x_{1}, \ldots, x_{l}\right) I^{n}=I^{n+1}$ for all $n$ sufficiently large. The ideal $J=\left(x_{1}, \ldots, x_{l}\right)$ is called a minimal reduction of $I$ and the smallest nonnegative integer $n$ satisfying $J I^{n}=I^{n+1}$ is denoted $r_{J}(I)$. The reduction number of $I$ is defined by

$$
r(I):=\min \left\{r_{J}(I) \mid J \text { a minimal reduction of } I\right\} .
$$

If $\operatorname{gr}_{I}(R)$ is Cohen-Macaulay and $I$ is equimultiple, then $r_{J}(I)=r(I)$ for any minimal reduction $J$ of $I([\mathrm{H}]$ or $[\mathrm{T}])$.

Suppose $R=\bigoplus R_{n}$ is a nonnegatively graded Noetherian ring. We denote by $R^{+}$the ideal of $R$ generated by all homogeneous forms of positive degree. Assume now that $R_{0}$ is local. Then $R$ has a unique homogeneous maximal ideal $M$. By [MR], $R$ is Cohen-Macaulay (abbreviated CM) if and only if $R_{M}$ is CM. We define depth $R$ to mean $\operatorname{depth}_{M} R$. That is, depth $R$ is the smallest nonnegative integer $s$ such that $H_{M}^{s}(R) \neq 0$, where $H_{M}^{i}(R)$ denotes the $i^{\text {th }}$ local cohomology module of $R$ with support in $M$. As a general reference on local cohomology, we refer the reader to $[\mathrm{Gr}]$ or $[\mathrm{GW}]$.

Let $A$ be a graded $R$-module. For $n \in \mathbf{Z}$, we let $A(n)$ denote the graded $R$-module $A$ twisted by $n$; i.e., $A(n)_{p}=A_{n+p}$ for all integers $p$. We note that if $A$ is graded and $I$ is a homogeneous ideal of $R$, then $H_{I}^{i}(A)$ is also a graded $R$-module and $H_{I}^{i}(A(n)) \cong H^{i}(A)(n)$ (as graded modules) for all $n$. If

$$
0 \longrightarrow A \longrightarrow B \longrightarrow C \longrightarrow 0
$$

is an exact sequence of graded $R$-modules with homogeneous (degree 0 ) maps, then the induced exact sequence on local cohomology

$$
\cdots \longrightarrow H_{I}^{i}(A) \longrightarrow H_{I}^{i}(B) \longrightarrow H_{I}^{i}(C) \longrightarrow \cdots
$$

also has homogeneous maps. If $A$ is finitely generated over $R$, then for each $i \geq 0$ $H_{M}^{i}(A)_{n}=0$ for $n$ sufficiently large. For $i \geq 0$, we define

$$
a_{i}(A):=\max \left\{n \in \boldsymbol{Z} \mid H_{M}^{i}(A)_{n} \neq 0\right\} .
$$

If $H_{M}^{i}(A)=0$ we set $a_{i}(A)=-\infty$.

Now suppose $x \in A_{k}$ is $A$-regular and let $s=\operatorname{depth} A$. Then from the short exact sequence 


$$
0 \longrightarrow A(-k) \stackrel{x}{\longrightarrow} A \longrightarrow A / x A \longrightarrow 0
$$

we obtain

$$
0 \longrightarrow H_{M}^{s-1}(A / x A)_{n} \longrightarrow H_{M}^{s}(A)_{n-k} \longrightarrow H_{M}^{s}(A)_{n}
$$

for all $n$. If $k \geq 1$ it is easy to see that

$$
a_{s-1}(A / x A)=a_{s}(A)+k .
$$

\section{Generalized depth}

Let $(R, m)$ be a local ring and $I$ an ideal of $R$. Using the language of [TI], $R$ is said to be a generalized Cohen-Macaulay ring with respect to $I$ if $H_{m}^{i}(R)$ is annihilated by some power of $I$ for all $i<\operatorname{dim} R$. (See also [Sch1], [CST] and [T1] in connection with generalized Cohen-Macaulay rings.) In this spirit, we define the generalized depth of $R$ with respect to $I$, abbreviated $g$-depth $R$, by

$$
\text { g-depth } R:=\sup \left\{k \in \mathbf{Z} \mid I \subseteq \sqrt{\operatorname{ann}_{R} H_{m}^{i}(R)} \text { for all } i<k\right\} .
$$

This number has previously been studied by both Brodmann [Br1] and Faltings $[\mathrm{F}]$ in their investigations of the finiteness properties of $H_{m}^{i}(R)$ (or more generally, of $H_{J}^{i}(M)$ for an ideal $J$ of $R$ and a finitely generated $R$-module $\left.M\right)$. The following proposition is a special case of Brodmann's result. As the proof is not long, we include it along with the statement.

For a Noetherian ring $R$ and an ideal $I$ of $R$, we let $\mathrm{D}(I)$ denote the subset of $\operatorname{Spec}(R)$ consisting of all prime ideals of $R$ not containing $I$.

Proposition 2.1 (cf. [Br1], Satz 3.12). Suppose that $(R, m)$ is a local ring which is the homomorphic image of a regular local ring and let $I$ be an ideal of $R$. Then

$$
\text { g-depth } R=\min _{p \in \mathrm{D}(I)}\left\{\operatorname{depth} R_{p}+\operatorname{dim} R / p\right\},
$$

where the right-hand side is defined to be $\infty$ if $\mathrm{D}(I)=\emptyset$.

Proof. For convenience, let $s(I)$ denote the right-hand side of the above equality. Let $R=S / J$ where $S$ is a $d$-dimensional regular local ring and let $E$ be the injective hull of the residue field of $S$. Then by local duality ([Ha]),

$$
H_{m}^{i}(R) \cong \operatorname{Ext}_{S}^{d-i}(R, S)^{*} \text { for all } i,
$$

where ${ }^{*}$ denotes the functor $\operatorname{Hom}_{S}(-, E)$. Since $\operatorname{ann}_{S} M=\operatorname{ann}_{S} M^{*}$ for all f.g. 
$S$-modules $M$, we see that some power of $I$ annihilates $H_{m}^{i}(R)$ if and only if some power of $I$ annihilates $\operatorname{Ext}_{S}^{d-t}(R, S)$.

Now let $p$ be a prime ideal of $R$ and $q$ a prime ideal of $S$ containing $J$ such that $q / J=p$. Let $r=\operatorname{dim} S_{q}$. Then

$$
d=\operatorname{dim} S=\operatorname{dim} S_{q}+\operatorname{dim} S / q=r+\operatorname{dim} R / p .
$$

Thus

$$
\begin{aligned}
\operatorname{Ext}_{S}^{d-i}(R, S)_{p}=0 & \Leftrightarrow \operatorname{Ext}_{S_{q}}^{d-i}\left(R_{p}, S_{q}\right)=0 \\
& \Leftrightarrow \operatorname{Ext}_{S_{q}}^{r-(i-\operatorname{dim} R / p)}\left(R_{p}, S_{q}\right)^{*}=0 \\
& \Leftrightarrow H_{p R_{p}}^{i-\operatorname{dim} R / p}\left(R_{p}\right)=0 .
\end{aligned}
$$

Suppose g-depth $R \geq k$. Then some power of $I$ annihilates $\operatorname{Ext}_{S}^{d-i}(R, S)$ for $i=0, \ldots, k-1$. Thus, $\operatorname{Ext}_{S}^{d-i}(R, S)_{p}=0$ for $0 \leq i \leq k-1$ and all $p \in$ $\mathrm{D}(I)$. Hence, $H_{p R_{p}}^{1-\operatorname{dim} R / p}\left(R_{p}\right)=0$ for $0 \leq i \leq k-1$ and all $p \in \mathrm{D}(I)$, and therefore $\operatorname{depth} R_{p} \geq k-\operatorname{dim} R / p$ for all $p \in \mathrm{D}(I)$. This proves that $\mathrm{g}$ - $\operatorname{depth}_{I} R$ $\leq s(I)$.

Conversely, suppose depth $R_{p}+\operatorname{dim} R / p \geq k$ for all $p \in \mathrm{D}(I)$. Then

$$
H_{p R_{p}}^{i-\operatorname{dim} R / p}\left(R_{p}\right)=0
$$

for $0 \leq i \leq k-1$ and all $p \in \mathrm{D}(I)$. Thus $\operatorname{Ext}_{S}^{d-i}(R, S)_{p}=0$ for $0 \leq i \leq k-1$ and all $p \in \mathrm{D}(I)$. Therefore

$$
I \subset \sqrt{\operatorname{ann}_{R}\left(\operatorname{Ext}_{S}^{d-i}(R, S)\right)}=\sqrt{\operatorname{ann}_{R} H_{m}^{i}(R)} \text { for } 0 \leq i \leq k-1 .
$$

This proves that $\mathrm{g}$-depth $R \geq s(I)$.

We will make use of the following fact:

Remark 2.2. Let $(R, m)$ be a catenary local ring and $p \subset q$ primes of $R$. Then

$$
\operatorname{depth} R_{q}+\operatorname{dim} R / q \leq \operatorname{depth} R_{p}+\operatorname{dim} R / p .
$$

Proof. Since $R$ is catenary, $\operatorname{dim} R / p-\operatorname{dim} R / q=$ ht $q / p$. Thus, by localizing at $q$ we may assume $q$ is the maximal ideal of $R$. By Ischebeck's Theorem ([Mat], Theorem 17.1), $\operatorname{Ext}^{2}(R / p, R)=0$ for $i<\operatorname{depth} R-\operatorname{dim} R / p$. Therefore $\operatorname{depth}_{p} R+\operatorname{dim} R / p \geq \operatorname{depth} R$.

Remark 2.3. Let $(R, m)$ be a local ring and $I$ an ideal of $R$. Then

(a) g-depth $R=$ g-depth $_{I \hat{R}} \hat{R}$ where $\hat{R}$ is the $m$-adic completion of $R$. 
(b) depth $R \leq$ g-depth $R$.

(c) g-depth $R \leq \operatorname{dim} R$ if and only if $I$ is not nilpotent.

Proof. The first statement follows from the fact that $H_{m \hat{R}}^{i}(\hat{R}) \cong H_{m}^{i}(R) \bigotimes_{R}$ $\hat{R}$. Part (b) is obvious from the definition of g-depth. For part (c), we may first assume that $R$ is complete so that $R$ is the homomorphic image of a regular local ring. If $g$-depth ${ }_{I}(R)<\infty$ then $\mathrm{D}(I) \neq \emptyset$, so $I$ is not nilpotent. Conversely, if $I$ is not nilpotent let $p$ be minimal prime not containing $I$. Then $g$-depth $R$ $\leq \operatorname{depth} R_{p}+\operatorname{dim} R / p \leq \operatorname{dim} R$.

Let $R=\bigoplus R_{n}$ be a nonnegatively graded Noetherian ring where $R_{0}$ is a local ring. For a homogeneous ideal $I$, we define the generalized depth of $R$ with respect to $I$ to be $g$-depth ${ }_{I R_{M}} R_{M}$ where $M$ is the homogeneous maximal ideal of $R$. In this case, it is clear that $\mathrm{g}$-depth $R \geq k$ if and only if some power of $I$ annihilates $H_{M}^{i}(R)$ for $i=0, \ldots, k-1$.

Remark 2.4. Let $R$ be a nonnegatively graded Noetherian ring such that $R_{0}$ is local and let $\hat{R}_{0}$ be the completion of $R_{0}$ with respect to its maximal ideal. Let $S=R$ $\otimes_{R_{0}} \hat{R}_{0}$ and $I$ a homogeneous ideal of $R$. Then g-depth ${ }_{I} R=$ g-depth $I S$.

Proof. Let $M$ be the unique homogeneous maximal ideal of $R$. Then $M S$ is the unique maximal homogeneous ideal of $S$ and $H_{M S}^{i}(S) \cong H_{M}^{i}(R) \bigotimes_{R_{0}} \hat{R}_{0}$ for all $i \geq 0$.

The proof of the following result can be found in [TI], although we state it here in a slightly stronger form.

Lemma 2.5 ([TI], Lemma 2.2). Let $R$ be a graded ring as in Remark 2.4. Then g-depth ${ }_{R+} R \geq k$ if and only if there exists an integer $t$ such that $H_{M}^{i}(R)_{n}=0$ for $n \leq t$ and $0 \leq i \leq k-1$.

\section{The depths of $R[I t]$ and $\operatorname{gr}_{I}(R)$}

In this section we prove several results which illustrate the relationship between depth $R[I t]$ and depth $\operatorname{gr}_{I}(R)$. We begin with an elementary lemma:

Lemma 3.1. Let $R$ be a local ring and $I$ an ideal of $R$. Let $S=R[I t]$ and $G=$ $\operatorname{gr}_{I}(R)$. Suppose $P$ is a prime ideal of $S$ which contains $I S$ but doesn't contain $S^{+}$. 
Then depth $S_{P}=$ depth $G_{Q}+1$, where $Q=P / I S$.

Proof. Choose $x \in I$ such that $x t \notin P$. Then $x S_{P}=I S_{P}$; for if $a \in I$, then $\frac{a}{1}=x \cdot \frac{a t}{x t} \in x S_{P}$. Therefore, $G_{Q} \cong S_{P} / x S_{P}$. Now if $x$ is a zero-divisor in $S_{P}$, then there exists an associated prime $q$ of $S$ such that $q \subset P$ and $x \in q$. But if $q=\left(0:_{s} f\right)$, then $x f=0$. Hence $x t f=0$ and so $x t \in q \subset P$, a contradiction. Thus $x$ is regular in $S_{P}$, which completes the proof.

We now prove a simple formula relating the generalized depths of $R[I t]$ and $\operatorname{gr}_{I}(R)$ (cf. [TI], Proposition 3.3). This formula will play a key role in the proofs that follow.

Proposition 3.2. Let $R$ be a local ring and $I$ and ideal of $R$. Let $S=R[I t]$ and $G=\operatorname{gr}_{I}(R)$. Then

$$
\text { g-depth } S_{+} S=\text { g-depth } \operatorname{de}_{+} G+1 \text {. }
$$

Proof. By Remark 2.4, we may assume $R$ is complete. Thus, $R$ is the homomorphic image of a regular local ring. Consequently, $S$ and $G$ are the homomorphic images of regular rings and so we may use Proposition 2.1.

Suppose g-depth ${ }_{S+} S \geq k$. Let $Q$ be a prime ideal of $G$ not containing $G^{+}$. Then there exists a prime $P \supseteq I S$ of $S$ such that $P / I S=Q$. Evidently, $P \in$ $\mathrm{D}\left(S^{+}\right)$. By Lemma 3.1 and Proposition 2.1,

$$
\operatorname{depth} G_{Q}+\operatorname{dim} G / Q=\operatorname{depth} S_{P}-1+\operatorname{dim} S / P \geq k-1 .
$$

Therefore, g-depth ${ }_{G+} G \geq$ g-depth $S_{+} S-1$.

Now suppose g-depth ${ }_{G+} G \geq k$. Let $p$ be a homogeneous prime ideal of $S$ not containing $S^{+}$. Then there exists $P \in \mathrm{D}\left(S^{+}\right)$such that $(I S, p) \subset P$. Otherwise, $S^{+} \subset \sqrt{(p, I S)}$ and so $I^{n} t^{n}=p_{n}+I^{n+1} t^{n}$ for $n$ sufficiently large. By Nakayama's lemma, this implies that $p_{n}=I^{n} t^{n}$ and so $S^{+} \subset p$, a contradiction. Now let $Q=P / I S$. Then

$$
\begin{aligned}
\operatorname{depth} S_{p}+\operatorname{dim} S / p & \geq \operatorname{depth} S_{P}+\operatorname{dim} S / P \quad(\text { Remark 2.2) } \\
& \geq \operatorname{depth} G_{Q}+1+\operatorname{dim} G / Q \quad(\text { Lemma 3.1) } \\
& \geq k+1 .
\end{aligned}
$$

If $p$ is not homogeneous then depth $S_{p}+\operatorname{dim} S / p=\operatorname{depth} S_{p^{*}}+\operatorname{dim} \mathrm{S} / p^{*}$, where $p^{*}$ is the ideal generated by homogeneous elements of $p$ (see [GW]). 
Thus, g-depth $S_{+} S \geq$ g-depth $G_{+} G+1$.

Lemma 3.3. Let $(R, m)$ be a local ring and $I$ an ideal of $R$. Let $S=R[I t]$ and $G=\operatorname{gr}_{I}(R)$. Then

(a) If depth $R<\operatorname{depth} S-1$ then depth $G=\operatorname{depth} R$.

(b) If $\operatorname{depth} R \geq \operatorname{depth} S-1$ then $\operatorname{depth} G \geq \operatorname{depth} S-1$.

Proof. Consider the following two exact sequences (cf. [Hu]):

$$
\begin{aligned}
& 0 \longrightarrow S^{+} \longrightarrow S \longrightarrow R \longrightarrow 0 \\
& 0 \longrightarrow I S \longrightarrow S \longrightarrow G \longrightarrow 0 .
\end{aligned}
$$

We view these two sequences in the catagory of graded $S$-modules with homogeneous maps, where $R$ is considered as a graded $S$-module concentrated in degree 0 .

Let $M$ and $N$ denote the homogeneous maximal ideals of $S$ and $G$, respectively. Let $t=\operatorname{depth} S$. Using (3.4) and the corresponding long exact sequence on local cohomology, we see that $H_{m}^{i}(R) \cong H_{M}^{i+1}\left(S^{+}\right)$for $i \leq t-2$. Likewise, using sequence (3.5) we get that $H_{N}^{i}(G) \cong H_{M}^{i+1}(I S)$ for $i \leq t-2$. Since $I S \cong S^{+}(1)$, we have $H_{M}^{i}(I S) \cong H_{M}^{i}\left(S^{+}\right)(1)$ for all $i$. Therefore $H_{N}^{i}(G) \cong H_{m}^{i}(R)(1)$ for $i \leq t$ -2 . Both (a) and (b) now follow.

It should be noted that both (a) and (b) are possible in the above lemma. Of course, (b) holds whenever $R$ is Cohen-Macaulay. As an example of case (a) occurring, consider the following example due to Goto and Shimoda ([GS1], Remark $3.5)$ : let $k[|x, y|]$ be a formal power series ring in two variables over a field $k, R$ the subring $k\left[\left|x^{2}, x y, y, x^{5}\right|\right]$ and $I$ the ideal $\left(x^{4}, y\right) R$. Then depth $R[I t]=3$ and $\operatorname{depth} R=1$.

We now show depth $\operatorname{gr}_{I}(R)$ can never exceed depth $R[I t]$.

Proposition 3.6. Let $(R, m)$ be a local ring, $I$ an ideal of $R, S=R[I t]$ and $G$ $=\operatorname{gr}_{I}(R)$. Then depth $G \leq \operatorname{depth} S$.

Proof. As before, let $M$ and $N$ denote the maximal homogeneous ideals of $S$ and $G$, respectively. Let $s=\operatorname{depth} G$. Using sequence (3.5) above, we obtain that

$$
H_{M}^{i}(I S)_{n} \cong H_{M}^{i}(S)_{n} \text { for } i \leq s-1 \text { and all } n \text {. }
$$

Applying the long exact sequence on local cohomology to (3.5), we have

$$
\cdots \longrightarrow H_{m}^{i-1}(R)_{n} \longrightarrow H_{M}^{i}\left(S^{+}\right)_{n} \longrightarrow H_{M}^{i}(S)_{n} \longrightarrow H_{m}^{i}(R)_{n} \longrightarrow \cdots
$$


for all $n$ and all $i$. But $H_{m}^{i}(R)_{n}=0$ for all $n \neq 0$, so

$$
H_{M}^{i}\left(S^{+}\right)_{n} \cong H_{M}^{i}(S)_{n} \text { for } i \geq 0 \text { and } n \neq 0 .
$$

Now since $I S \cong S^{+}(1)$, we have

$$
H_{M}^{i}(I S)_{n} \cong H_{M}^{i}\left(S^{+}\right)_{n+1} \text { for all } i, n \text {. }
$$

Combining (3.7), (3.8) and (3.9), we obtain that $H_{M}^{i}(S)_{n} \cong H_{M}^{i}(S)_{n+1}$ for $n \neq-1$ and $i \leq s-1$. But $H_{M}^{i}(S)_{n}=0$ for $n$ sufficiently large, so $H_{M}^{i}(S)_{n}=0$ for $n \geq 0$. On the other hand, Proposition 3.2 implies that g-depth $S_{+} S=$ g-depth $\operatorname{de}_{G+} G+1$ $\geq s+1$. So by Lemma $2.5, H_{M}^{i}(S)_{n}=0$ for $n$ sufficiently small and $i \leq s$. Hence $H_{M}^{i}(S)_{n}=0$ for $n \leq-1$ and $i \leq s-1$. Therefore, $H_{M}^{i}(S)=0$ for $i \leq s-1$ and so depth $S \geq s$.

Theorem 3.10. Let $(R, m)$ be a local ring and $I$ an ideal of $R$. Let $S=R[I t]$ and $G=\operatorname{gr}_{I}(R)$. Suppose depth $G<\operatorname{depth} R$. Then

$$
\operatorname{depth} S=\operatorname{depth} G+1 \text {. }
$$

Proof. By Lemma 3.3 and Proposition 3.6, we have

$$
\text { depth } G \leq \operatorname{depth} S \leq \operatorname{depth} G+1 \text {. }
$$

Let $s=\operatorname{depth} G$. From (3.5) we obtain

$$
0 \longrightarrow H_{M}^{s}(I S)_{n} \longrightarrow H_{M}^{s}(S)_{n} \longrightarrow H_{N}^{s}(G)_{n}
$$

for all $n$. Using (3.4) and the fact that $s<\operatorname{depth} R$ we get that $H_{M}^{s}\left(S^{+}\right)_{n} \cong$ $H_{M}^{s}(S)_{n}$ for all $n$. Since $H_{M}^{s}(I S)_{n} \cong H_{M}^{s}\left(S^{+}\right)_{n+1}$ for all $n$ (see (3.9) above), we get

$$
0 \longrightarrow H_{M}^{s}(S)_{n+1} \longrightarrow H_{M}^{s}(S)_{n}
$$

for all $n$. But g-depth $S_{+} S=$ g-depth ${ }_{G+} G+1 \geq s+1$, so $H_{M}^{s}(S)_{n}=0$ for $n$ sufficiently small by Lemma 2.5 . Therefore $H_{M}^{s}(S)=0$ and depth $S \geq s+1$.

As a special case of this theorem, we get the following generalization of (1.4):

Corollary 3.12. Let $(R, m)$ be a $C M$ local ring and $I$ an ideal of $R$. Suppose $\operatorname{gr}_{I}(R)$ is not $C M$. Then

$$
\operatorname{depth} R[I t]=\operatorname{depth} \operatorname{gr}_{I}(R)+1 \text {. }
$$

Reformulating (1.3) in the case $R$ is CM, $R[I t]$ is $C M$ if and only if $\operatorname{gr}_{I}(R)$ is 
$\mathrm{CM}$ and $a_{d}\left(\operatorname{gr}_{I}(R)\right)<0$. The next theorem generalizes one direction of this result.

Theorem 3.13. Let $(R, m)$ be a local ring, $I$ an ideal, $S=R[I t]$ and $G=$ $\operatorname{gr}_{I}(R)$. Let $s=\operatorname{depth} G$. If $a_{s}(G)<0$ then

$$
\operatorname{depth} S \geq \operatorname{depth} G+1 \text {. }
$$

Proof. By Proposition 3.6, we know that $\operatorname{depth} S \geq \operatorname{depth} G=s$. From (3.4) we get that

$$
H_{m}^{s-1}(R)_{n} \longrightarrow H_{M}^{s}\left(S^{+}\right)_{n} \longrightarrow H_{M}^{s}(S)_{n} \longrightarrow H_{m}^{s}(R)_{n}
$$

for all $n$. Therefore $H_{M}^{s}\left(S^{+}\right)_{n} \cong H_{M}^{s}(S)_{n}$ for $n \neq 0$. Using (3.5), we obtain

$$
0 \longrightarrow H_{M}^{s}(I S)_{n} \longrightarrow H_{M}^{s}(S)_{n} \longrightarrow H_{N}^{s}(G)_{n}
$$

for all $n$. As $H_{M}^{s}(I S)_{n} \cong H_{M}^{s}\left(S^{+}\right)_{n+1}$ for all $n$ by (3.9), we get that

$$
0 \longrightarrow H_{M}^{s}(S)_{n+1} \longrightarrow H_{M}^{s}(S)_{n}
$$

for $n \neq-1$. By Proposition 3.2, g-depth ${ }_{S+} S=\mathrm{g}$ - $\operatorname{depth}_{G+} G+1 \geq s+1$, so $H_{M}^{s}(S)_{n}=0$ for $n$ sufficiently small. Hence $H_{M}^{s}(S)_{n}=0$ for $n \leq-1$.

As $a_{s}(G)<0, H_{M}^{s}(G)_{n}=0$ for $n \geq 0$. Thus, from (3.14) we have that $H_{M}^{s}(I S)_{n} \cong H_{M}^{s}(S)_{n}$ for $n \geq 0$. Hence, for $n \geq 0$

$$
H_{M}^{s}(S)_{n+1} \cong H_{M}^{s}\left(S^{+}\right)_{n+1} \cong H_{M}^{s}(I S)_{n} \cong H_{M}^{s}(S)_{n} .
$$

Since $H_{M}^{s}(S)_{n}=0$ for $n$ sufficiently large, we see that $H_{M}^{s}(S)_{n}=0$ for $n \geq 0$. Hence $H_{M}^{s}(S)=0$ and depth $S \geq s+1$.

In light of Lemma 3.3, we see that we get equality in the above theorem if depth $G \neq \operatorname{depth} R$.

We remark that the converse to this theorem is false if $s<\operatorname{dim} R$. For example, let $R=k\left[x^{4}, x^{3} y, x y^{3}, y^{4}\right]$ where $k$ is any field, and let $I=\left(x^{4}, x^{3} y, y^{4}\right) R$. Using Bayer and Stillman's Macaulay, one finds that depth $R[I t]=2$ and

$$
\operatorname{gr}_{I}(R) \cong k[a, b, c, d] /\left(a c, a b^{2}, a^{2} b, a^{3}, c^{4}-b^{3} d\right)
$$

where $a, b, c, d$ are indeterminates with $\operatorname{deg} a=0$ and $\operatorname{deg} b=\operatorname{deg} c=\operatorname{deg} d$ $=1$. It is easy to see that $d$ is regular on $G=\operatorname{gr}_{I}(R)$ and that $a_{0}(G / d G)=1$. Thus depth $\operatorname{gr}_{I}(R)=1$ and $a_{1}\left(\operatorname{gr}_{I}(R)\right)=0$ by $(1.5)$.

As an example of a situation where Theorem 3.13 can be applied, consider the following 
Proposition 3.15. Let $(R, m)$ be a local Buchsbaum ring and $I$ an ideal generated by a system of parameters. Let $G=\operatorname{gr}_{I}(R)$ and $s=\operatorname{depth} G$. Then $a_{s}(G)=-s$.

Proof. First note that if $\operatorname{dim} R=0$ then $I=0$ and the theorem holds. Thus we may assume $R$ is of positive dimension. By passing to $R(X)=R[X]_{m R[X]}$ we may assume that the residue field of $R$ is infinite. (The fact that $R(X)$ is still Buchsbaum is the content of Lemma 2.26 of [SV].)

Choose generators $x_{1}, \ldots, x_{d}$ (where $d=\operatorname{dim} R$ ) for $I$ such that the images of $x_{1}, \ldots, x_{s}$ in $G_{1}$ form a regular sequence in $G$. Let $T=R /\left(x_{1}, \ldots, x_{s}\right)$ and $J=$ $\left(x_{s+1}, \ldots, x_{d}\right) T$. Then $T$ is also a Buchsbaum ring and $\operatorname{gr}_{J}(T) \cong G /\left(x_{1}^{*}, \ldots, x_{s}^{*}\right)$. By $(1.5) a_{s}(G)=a_{0}\left(G /\left(x_{1}^{*}, \ldots, x_{s}^{*}\right)\right)-s$, so it is enough to prove the proposition in the case $s=0$.

So suppose depth $G=0$. Let $a^{\prime} \in H_{N}^{0}(G)_{n}$ for some $n \geq 1$ and choose $a \in$ $I^{n}$ whose image in $I^{n} / I^{n+1}$ is $a^{\prime}$. We will show $a \in I^{n+1}$ and so $a^{\prime}=0$. There exists a sufficiently large integer $k$ such that $a^{\prime} G_{k}=0$; i.e., $a I^{k} \subset I^{k+n+1}$. Thus, $a$ $\in\left(I^{k+n+1}: I^{k}\right) \cap I^{n}$. But by Lemma 1.15 of [SV], $\left(I^{k+1}: I\right) \cap I=I^{k}$ for $k \geq 1$. Using induction on $k$ and the fact that $\left(I^{k+n+1}: I^{k}\right)=\left(\left(I^{k+n+1}: I\right): I^{k-1}\right)$, we get that

$$
\left(I^{k+n+1}: I^{k}\right) \cap I=I^{n+1}
$$

for all $k, n \geq 1$. Hence $a \in I^{n+1}$, and so $H_{N}^{0}(G)_{n}=0$ for $n \geq 1$. Therefore, $a_{0}(G)=0$.

By $[G]$, for any pair of nonnegative integers $s \leq d$ there exist $d$-dimensional Buchsbaum rings with depth $s$. Thus, by (1.1) we see that for an arbitrary Buchsbaum ring $R$ it is not necessarily the case that $R[I t]$ is $\mathrm{CM}$ for all parameter ideals $I$. However, we do get the following result, which is a consequence of Theorem 3.13 and Proposition 3.15.

Corollary 3.16. Let $(R, m)$ be a local Buchsbaum ring of positive depth and $I$ an ideal of $R$ generated by a system of parameters. Then

$$
\text { depth } R[I t] \geq \text { depth } \operatorname{gr}_{I}(R)+1 \text {. }
$$

Of course if depth $R=0$ then depth $\operatorname{gr}_{I}(R)=\operatorname{depth} R[I t]=0$.

Note that we do not necessarily have equality in the above corollary. For instance, take $R$ to be any two-dimensional Buchsbaum local domain which is not CM (e.g. $k\left[\left|x^{4}, x^{3} y, x y^{3}, y^{4}\right|\right]$ ) and $I$ any ideal of $R$ generated by a system of pa- 
rameters. Then $R[I t]$ is CM by (1.1) but depth $\operatorname{gr}_{I}(R)=\operatorname{depth} R=1$ by Lemma 3.3 (a).

We also remark that Corollary 3.16 does not hold for arbitrary local rings. For if $R=k[|x, y, z|] /\left(x^{2}, x y^{2}\right)$ and $I=(y, z) R$ then one finds (using Macaulay, for instance) that depth $R[I t]=\operatorname{depth} \operatorname{gr}_{I}(R)=\operatorname{depth} R=1$.

\section{REFERENCES}

[Br1] M. Brodmann, Einige Ergebnisse aus der lokalen Kohomologietheorie und ihre Anwendung, Osnabrücker Schrifter zur Mathematik, 5 (1983).

[Br2] - Local cohomology of certain Rees- and form-rings I, J. Algebra, 81 (1984), $29-57$.

[Br3] - Local cohomology of certain Rees- and form-rings II, J. Algebra, 86 (1985), 457-493.

[CST] N. T. Cuong, P. Schenzel and N. V. Trung, Verallgemeinerte Cohen-MacaulayModuln, Math. Nachr., (1978), 57-73.

[F] G. Faltings, Über die Annulatoren lokaler Kohomologiegruppen, Arch. Math., 30 (1978), 473-476.

[G] S. Goto, On Buchsbaum rings, J. Algebra, 67 (1980), 272-279.

[GS1] S. Goto and Y. Shimoda, On Rees algebras over Buchsbaum rings, J. Math. Kyoto Univ., 20 (1980), 691-708.

[GS2] S. Goto and Y. Shimoda, On the Rees algebra of Cohen-Macaulay local rings, Commutative Algebra: analytical methods, Lecture Notes in Pure and Applied Math., no. 68, Dekker, New York, 1982.

[GW] S. Goto and K. Watanabe, On graded rings I, J. Math. Soc. Japan, 30 (2) (1978), $179-213$.

[Gr] A. Grothendieck (notes by R. Hartshorne), "Local cohomology," Lect. Notes in Math., 41, Springer-Verlag, Berlin, 1967.

[Ha] R. Hartshorne, "Residues and duality," Lect. Notes in Math., 20, Springer-Verlag, Berlin, 1966.

[H] S. Huckaba, Reduction numbers for ideals of higher analytic spread, Math. Proc. Cambridge Phil. Soc., 102 (1987), 49-57.

[HM] S. Huckaba and T. Marley, Depth properties of Rees algebras and associated graded rings, J. Algebra, 156, (1993) 259-271.

[Hu] C. Huneke, On the associated graded ring of an ideal, Ill. J. Math., 26 (1982), 121-137.

[Mat] H. Matsumura, "Commutative ring theory," Cambridge University Press, Cambridge, 1980.

[MR] J. Matijevic and P. Roberts, A conjecture of Nagata on graded Cohen-Macaulay rings, J. Math. Kyoto Univ., 14 (1974), 125-128.

[NR] D. G. Northcott and D. Rees, Reductions of ideals in local rings, Proc. Cambridge Phil. Soc., 50 (1954), 145-158.

[Sch1] P. Schenzel, Einige Anwendungen der lokalen Dualität und verallgemeinerte Cohen-Macaulay-Moduln, Math. Nachr., 69 (1975), 227-242.

[Sch2] - Regular sequences in Rees and symmetric algebras I, Manuscripta Math., 35 (1981), 173-193. 
[Sch3] - Regular sequences in Rees and symmetric algebras II, Manuscripta Math., 35 (1981), 331-341.

[SV] J. Stückrad and W. Vogel, "Buchsbaum rings and applications," Springer-Verlag, Berlin, 1986.

[T1] N. V. Trung, Toward a theory of generalized Cohen-Macaulay modules, Nagoya Math. J., 102 (1986), 1-49.

[T2] - Reduction exponent and degree bound for the defining equations of graded rings, Proc. Amer. Math. Soc., 101 (2) (1987), 229-236.

[TI] N. V. Trung and S. Ikeda, When is the Rees algebra Cohen-Macaulay?, Comm. Algebra, 17 (12) (1989), 2893-2922.

[V] G. Valla, Certain graded algebras are always Cohen-Macaulay, J. Algebra, 42 (1976), 537-548.

S. Huckaba

Department of Mathematics

Florida State University

Tallahassee, FL 32306-3027, U.S.A.

T. Marley

Department of Mathematics and Statistics

University of Nebraska

Lincoln, NE 68588-0323, U.S.A. 